\title{
Coordination of back bending and leg movements for quadrupedal locomotion
}

\author{
Baxi Zhong*, Yasemin Ozkan Aydin ${ }^{\dagger}$, Chaohui Gong*, Guillaume Sartoretti*, Yunjin $\mathrm{Wu}^{*}$, Jennifer Rieser ${ }^{\dagger}$, \\ Haosen Xing*, Jeffery Rankin ${ }^{\ddagger}$, Krijn Michel ${ }^{\S}$, Alfredo Nicieza ${ }^{\S}$, \\ John Hutchinson ${ }^{\uparrow}$, Daniel Goldman ${ }^{\dagger}$ and Howie Choset ${ }^{*}$ \\ *Carnegie Mellon Institution \\ ${ }^{\dagger}$ Georgia Institute of Technology \\ $\ddagger$ Rancho Research Institute \\ $\S$ Royal Veterinary College \\ IUniversity of Oviedo
}

\begin{abstract}
Many quadrupedal animals use lateral degrees of freedom in their backs to assist locomotion. This paper seeks to use a robotic model to demonstrate that back bending assists not only forward motion, but also lateral and turning motions. We present a simple planner that uses geometric mechanics to prescribe gaits that coordinate both leg movements and back bending motion. Using these geometric tools, we show that back-bending can improve stride displacement in the forward, rotational, and lateral directions. Interestingly, we observe that the animal's (salamander, Salamandra salamandra) back-bending is close to our calculated back-bending to improve forward displacement. In addition to locomotion performance improvement, back bending can also expand the target position space a robot can reach within one gait cycle. Our results are verified by conducting experiments with a robot moving on granular materials.
\end{abstract}

\section{INTRODUCTION}

A variety of quadrupedal animals have been observed to bend their backs and coordinate with their leg movements to improve locomotive performance [50, 35, 20, 14] while there is less research on robots with actuated back. Recent work in geometric mechanics [39, 38, 48, 55], has offered a framework to study many undulatory limbless locomotor behaviors [1, 6, 30, 41]. In this paper, we seek to design gaits to coordinate back bending to leg movements to improve the locomotive performance in forward, rotational and lateral directions. We formulate the back-bending quadrupedal locomotion as gait design on cylindrical shape space by reducing leg movements to one dimension. In doing so, we are able to extend geometric mechanics to study legged system, as well as to design and optimize gaits that improve locomotive capabilities and expand the locomotive reach of a legged robot. To the best of the authors' knowledge, there has been no research applying geometric mechanics to legged systems.

This paper shows that the use of the internal degree of freedom in the back actually improves locomotion, when compared with the same locomotion while keeping the back bending degree of freedom fixed, say at a "zero" position. Specifically, we show that properly coordinating the degree of freedom in the back increases the displacement per gait cycle, whether it be forward, lateral or rotational. The motion on
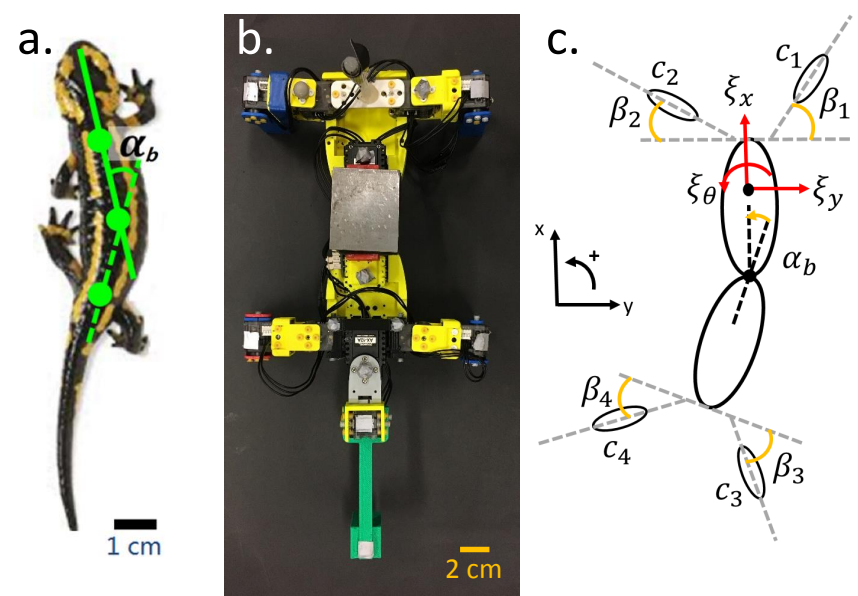

Fig. 1. The animal, robot and theoretical model. (a) top view of a Fire salamander (Salamandra salamandra). Body angle, $\alpha_{b}$, is defined as an angle between the center lines that are parallel to front and back part of the body. (b) top view of the robophysical model. It has two body parts connected with a servo, four 2 DoF legs and a tail. The metal part at the center is used to pick up the robot with an electromagnetic gripper. All legs and tail have same foot geometry (24X24 mm cube shape). (c) the theoretical model with shape variables and body velocities labeled.

the internal degree of freedom in the back can also expand the target position space a robot can reach within one gait cycle. Furthermore, forward motion gaits we calculated from geometric mechanics are very close to the back-bending gaits used by Fire salamander (Salamandra salamandra (Fig. 117)).

The paper is structured as follows: Section II provides an overview of prior work; Section III introduces geometric mechanics and its extension to cylindrical shape space; Section IV] introduces our robot; Section $\mathrm{V}$ details our numerical and experimental results. We finally discuss the significance of our work in section VI. Finally, we suggest that the reader go through the figures of this paper first, read the captions, and then read the text.

\section{RELATED WORK}

\section{A. Leg movement}

The coordination of back bending and leg movement for quadrupedal locomotion in this work extends existing literature 

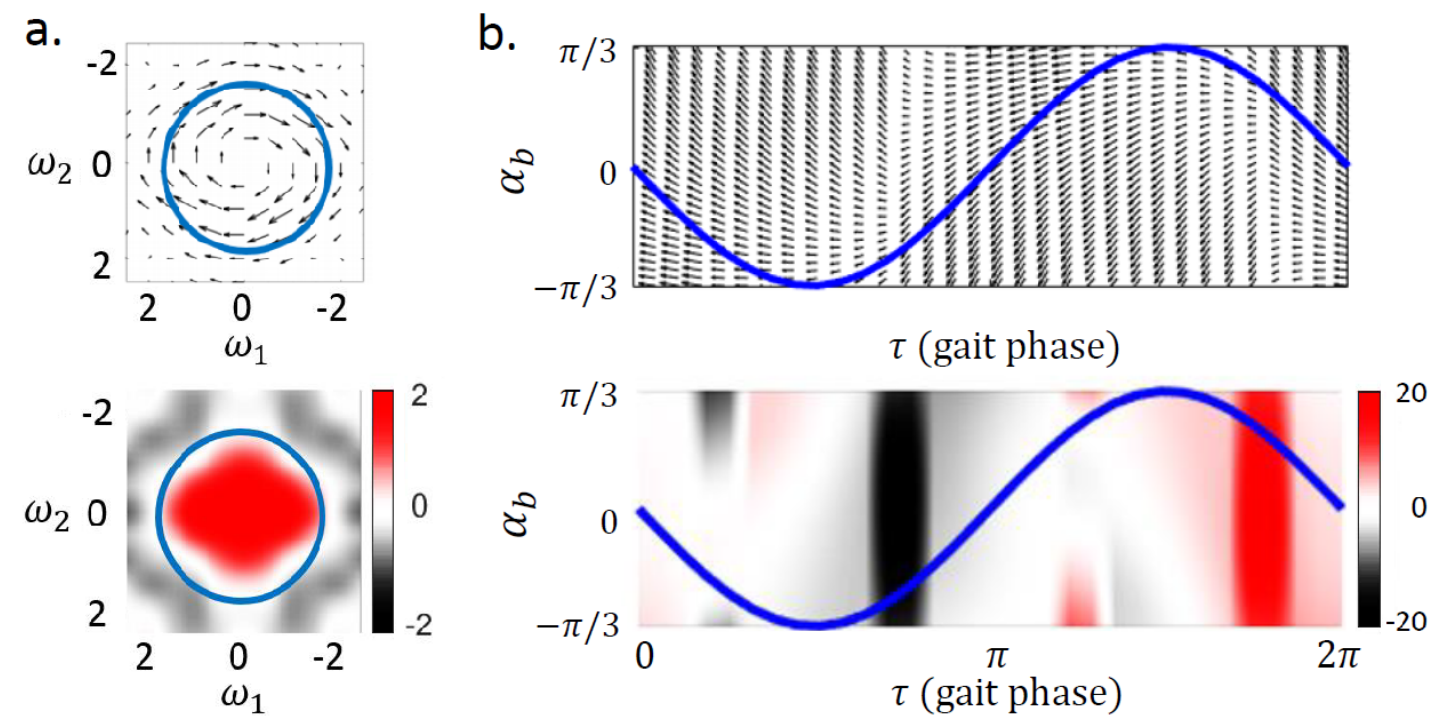

Fig. 2. The vector field and height functions in the reduced shape space for two different systems. (a) empirical connection vector field (top) and height function (bottom) corresponding to motions in the forward direction of an 8- link snake robot slithering on the surface of $6 \mathrm{~mm}$ plastic particles in Euclidean shape space. The blue circle represent the optimal gait in the corresponding shape space. (b) the connection vector field (top) and height function (bottom) corresponding to the forward motion of a quadruped robot moving with four beat walking gait on the surface of $\sim 1$ mm poppy seeds in cylindrical shape space. The blue curves represent a sample gait in the corresponding shape space. Red, white and black indicate positive, zero and negative values respectively.

on quadrupedal leg movements. We provide a brief review of literature on leg movements.

Foot placement determines a sequence of locations on the ground where a system places its feet. It may optimize criteria like stability or redundancy when applied to multi-legged locomotion. Mcghee and Iswandhi [40] introduced a heuristic gait planning algorithm for legged robots to maximize stability margin and minimize kinematic margin (the distance that the foothold of a given leg can travel in the opposite direction of motion before reaching the boundary of its workspace). Although this algorithm was adequate for hexapods, foot placements are not as well suited to quadrupeds because quadrupeds have more strict stability criterion [5]. Bai et al. [5] applied a similar approach to quadrupeds, which took lateral sequence walk (leg lifting follows the sequence: left hind leg, left front leg, right hind leg, right front leg [24]), as a primary gait then adapted it to the environment. These ideas were applied to the Little Dog platform [34, 57], where they planned the footsteps across the terrain and implemented them in presence of disturbance.

Many machine learning tools and algorithms have been used to determine and optimize gaits. Kim and Uther [31] applied Powell's minimization method [44] to design periodic footfall patterns for quadrupedal locomotion that were faster and more stable than previous hand-optimized gaits in RoboCup soccer competition. Kohl and Stone [33] applied the policy gradient method to design locus based [53] quadruped gaits and improved locomotion speed. Chernova and Veloso [11] applied the gradient-free evolutionary approach to quadruped gait learning, which relaxed the constrain of locus based search space. Degrave et al. [17] improved the learning algorithm by applying transfer learning, which shows knowledge sharing between gaits can greatly improve learning performance across different environments.
Biomimetics derives direct inspirations from biological systems. Inspired by cockroach racing over the rough surface, Saranli et al. [47] showed that Rhex, a hexapod robot [8, 9, 3], exhibited the ability for forward locomotion in uneven terrain and in the presence of obstacles. Inspired by the self-stabilizing ability of insects, Cham et al. [10] developed a stride period adaptation method for hexapod locomotion. Based on the important criteria (for example, minimization of unwanted displacements) identified by Alexander [2] for bipedal and quadrupedal animals, Smith and Poulakakis [51] compared phase relationships in the leg touchdown pattern of rotary gallop gait for quadrupedal robot and animals.

\section{B. Central pattern generator}

Inspired by analogous ideas from biology, Central Pattern Generators (CPGs) produce periodic signals to drive joint trajectories in shape space for locomotion or other repetitive tasks [26, 46]. CPGs have been extensively studied for quadrupedal locomotion [18, 54, 42, 28, 15, 27, 14]. We provide a brief review of $\mathrm{CPG}$ research line on quadrupeds.

Owaki et al. [42] investigated the mechanisms of inter-limb coordination of a quadrupedal robot from a CPG perspective. Ijspeert et al. [28] showed that CPGs can produce body-limb coordinated movements for the locomotion of a salamander robot, as well as generate gait transitions as commanded. Cruse [15] showed that stable gaits of arthropods can be generated with indirect coupling through sensory feedback and mechanical coupling. Finally, Crespi et al. [14] demonstrated that the body-limb coordination used by salamanders optimizes its locomotion speed.

\section{Turning motion}

While turning has been less studied, some progress has been made. Bien et al. [7] first introduced footfall planning for stable turning motions. Cho et al. [12] introduced the footfall 


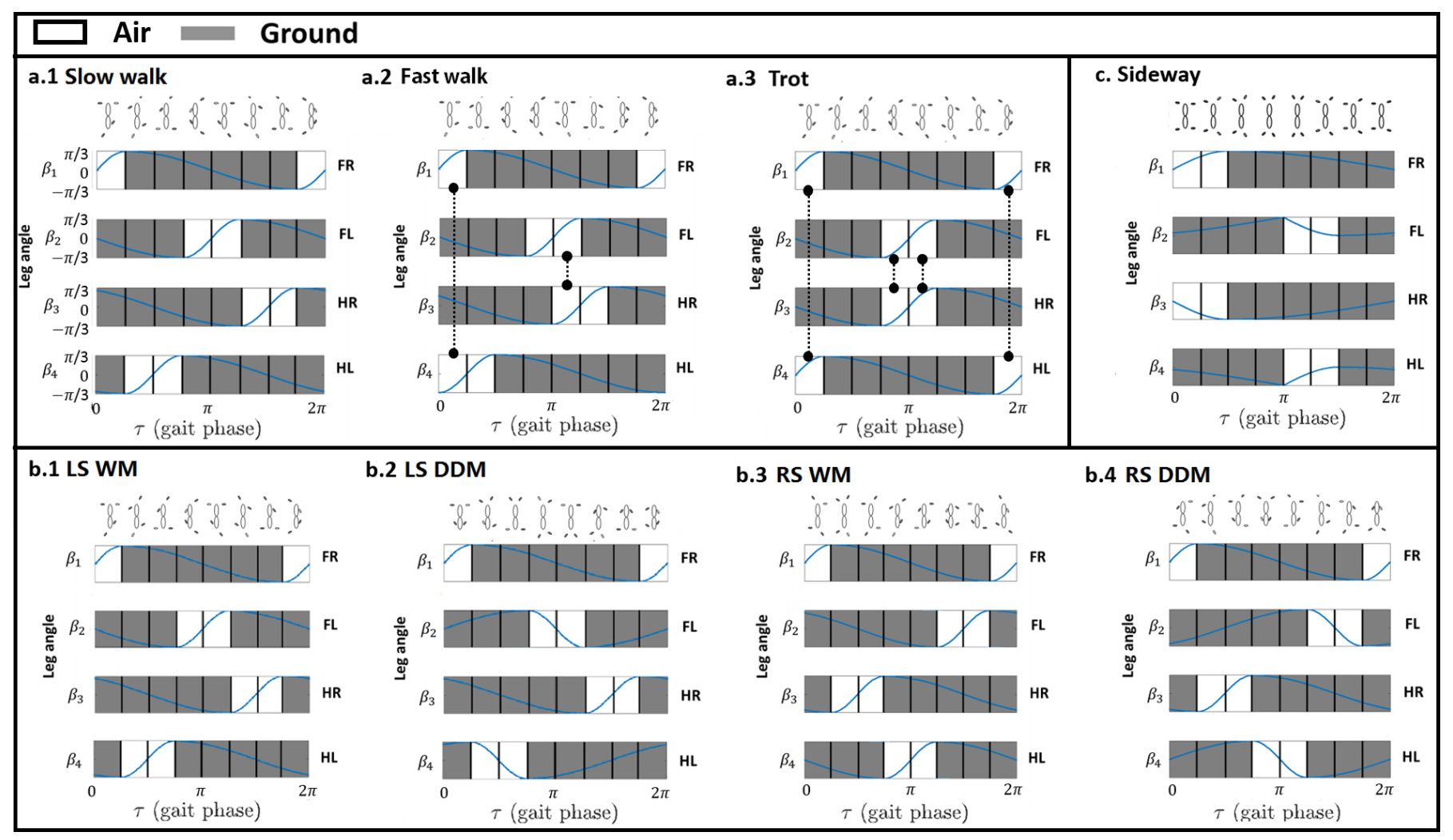

Fig. 3. The leg movement prescribed by gait phase $\tau$ for (a.1) (forward) trot, (a.2) (forward) fast walk, (a.3) (forward) slow walk (b.1) (rotational) lateral sequence walking machine (LS WM), (b.2) (rotational) lateral sequence differential drive machine (LS DDM), (b.3) (rotational) rotary sequence walking machine (RS WM), (b.4) (rotational) rotary sequence differential drive machine (RS DDM) and (c) lateral gaits. The cartoon sequence shows the leg joint angles and contact states at different gait phase. The dashed line in (a) indicate the overlaps of diagonal legs activation. The joint-angle curves as a function of gait phase are listed with stance phase indicated by the shading. The initials F, H, L, R stand for front, hind, left and right leg. All the panels have the same ordinate range as in (a.1).

planning objective function where speed, stability, translation direction and turning were all included. Kafkafi and Golani [29] showed body shapes are different for different motion patterns, specifically the body should be $S$-shaped for forward motion and $C$-shaped for turning motion. Palmer and Orin [43] designed a turning fuzzy controller by placing the fore legs to the outside of the turn and the hind legs to the inside.

\section{Geometric Mechanics}

Geometric mechanics provides the foundation of the work in this paper. In this section, we provide an overview of geometric mechanics and then extend geometric mechanics tools to legged systems with periodic ground contact. Using these tools, we numerically calculate a height function using granular resistive force theory. We finally use the height function to visually design a gait for a quadruped.

\section{A. Geometric mechanics overview}

We provide a concise overview of the geometric tools needed for this paper, but for a more detailed and comprehensive review, we refer readers to [1, 6, 16, 30, 38, 39, 41 , 48, 55, 22]

1) Kinematic reconstruction equation: In principally kinematic systems, where there is no drift in the system, the equations of motion reduce to

$$
\xi=A(r) \dot{r}
$$

where $\boldsymbol{\xi}=\left[\begin{array}{lll}\xi_{x} & \xi_{y} & \xi_{\theta}\end{array}\right]^{T} \in g$ denotes the body velocity in forward, lateral and rotational direction, $r$ denotes the shape variables (joint angles), and $\boldsymbol{A}(\boldsymbol{r})$ is the local connection, a matrix that relates shape velocity $\dot{\boldsymbol{r}}$ to body velocity $\boldsymbol{\xi}$. The Eq.(1) is also called the kinematic reconstruction equation. It maps the changes in internal shape variables (joint angles) to changes in position variables (position and orientation) of the robot.

2) Connection vector fields and height functions: Each row of the the connection corresponds to a component direction of the body velocity and therefore gives ride to a connection vector field, where for each shape, the corresponding vector denotes the maximal input set for that row's component direction. (Fig. 2). The body velocities in the forward, lateral and rotational directions are respectively computed as the dot product between connection vector fields and the shape velocity $\dot{\boldsymbol{r}}$. A shape velocity $\dot{\boldsymbol{r}}$ along the direction of the vector field would yield the largest possible body velocity in that direction, while a shape velocity $\dot{\boldsymbol{r}}$ orthogonal to the field would produce zero body velocity.

A gait is represented as a path in the shape space with the same start and end shape. Fig. 2 a shows a typical vector field 


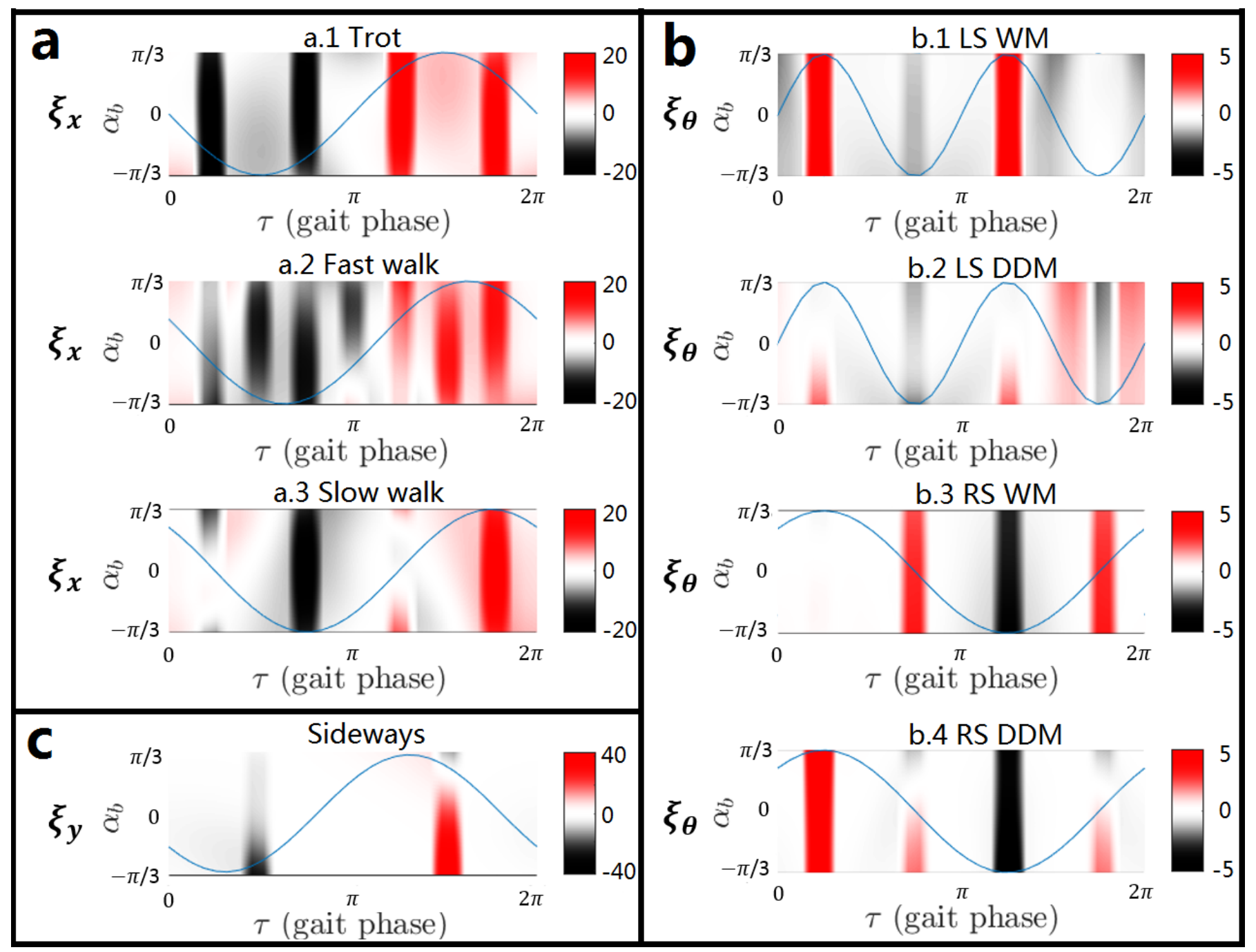

Fig. 4. Height function of (a) forward gaits (trot (top), fast walk (middle) and slow walk (bottom)) (b) turning gaits (lateral sequence walking machine (LS WM) (b1), lateral sequence differential drive machine (LS DDM) (b2), rotary sequence walking machine (RS WM) (b3) and rotary sequence differential drive machine (RS DDM) (b4)) and (c) sideways gaits in the reduced shape space. The blue curve is the identified "optimal" gait path. Red, white and black indicate positive, zero and negative values respectively. The $\mathrm{x}$-axis is same for all panels.

for limbless locomotion in Euclidean shape space, where gaits appear as closed loops. The line integral

$$
\left(\begin{array}{c}
\Delta x \\
\Delta y \\
\Delta \theta
\end{array}\right)=\int_{\partial \phi} \boldsymbol{A}(\boldsymbol{r}) \boldsymbol{r},
$$

can approximate the displacement resulting from a gait $\partial \phi$.

According to Stokes' Theorem, the line integral along a closed curve $\partial \phi$ is equal to the surface integral of the curl of $\boldsymbol{A}(\boldsymbol{r})$ over the area enclosed by $\partial \phi$ :

$$
\int_{\partial \phi} \boldsymbol{A}(\boldsymbol{r}) \boldsymbol{r}=\iint_{\phi} \boldsymbol{\nabla} \times \boldsymbol{A}(\boldsymbol{r}) r_{1} r_{2}
$$

where $\phi$ denotes the area enclosed by $\partial \phi$. The curl of the connection vector field $\nabla \times A(r)$ is referred to as the height function (Fig. 2a). The three rows of the vector field $A(r)$ can thus produce three height functions, in the forward, lateral and rotational direction respectively.

With the above derivation, we simplify the gait design for limbless locomotion to drawing a closed loop in Euclidean shape space. The displacements is approximated by the volume enclosed by the gait path. For example, in Fig. 2 a, drawing a circle with most volume enclosed represents designing gaits with largest forward stride displacement.

Often, a shape space can have some cyclic structure, as we will be the case for the legged systems examined in this paper. The shape space $\left(S^{1} \times R^{1}\right)$ can then be cylindrical [32] (Fig. 2p). Concurrent work has shown that the displacement is either the surface integral below or above the path. We refer reader to [22] for detailed derivation and proof of motion planning in cylinder shape spaces (this work also includes torus shape spaces). In this paper, we calculate the displacement as the surface integral underneath the path.

Similarly, we simplify the gait design problem for legged system to drawing a path in cylindrical shape space. For example, in Fig. 4, drawing a path with most volume underneath represents a gait with most forward displacement. 

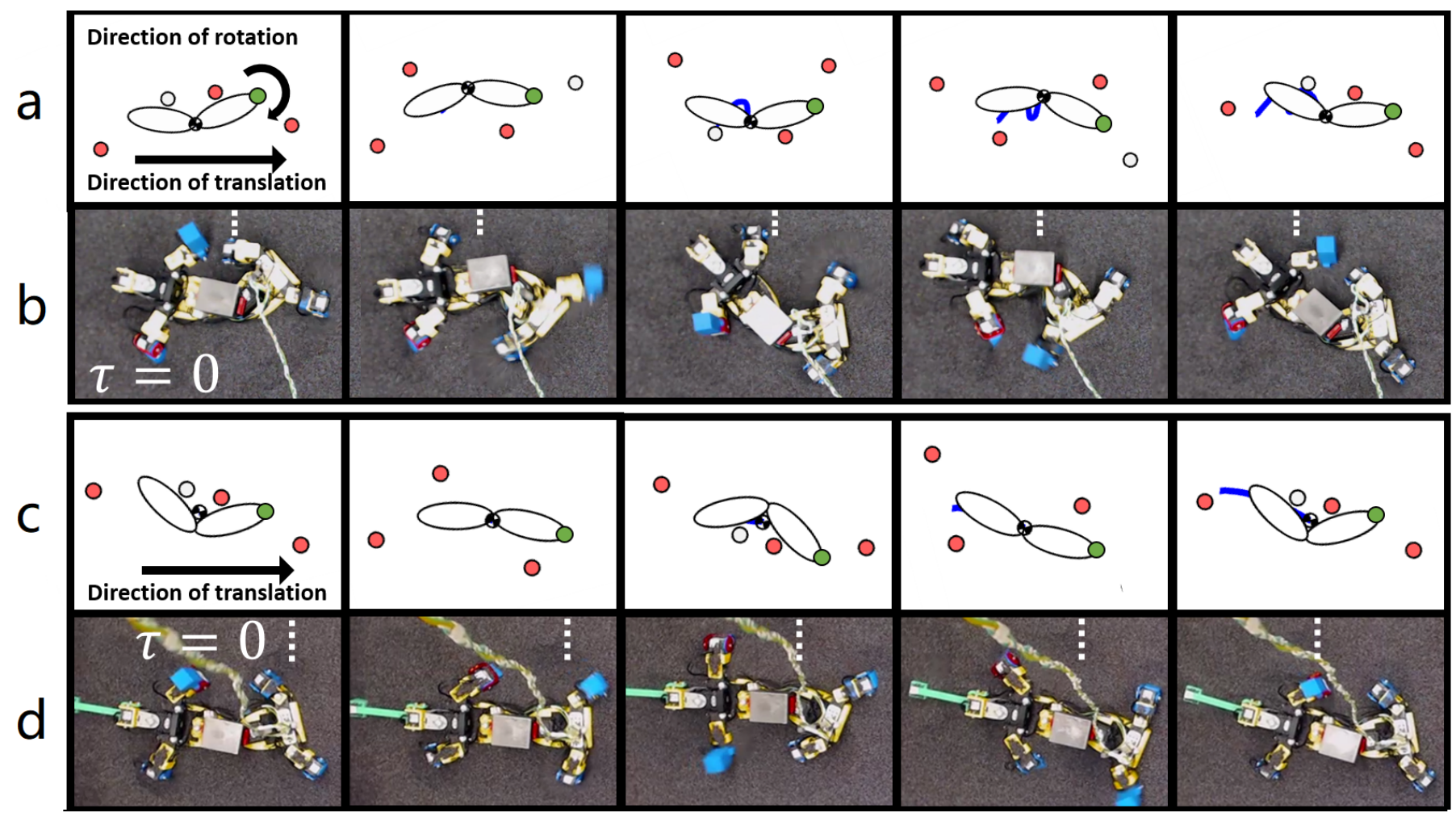

Fig. 5. The back bending coordination sequence with leg movement to turn head orientation (a and b) and increase forward displacement (c and d) for both simulation ( $a$ and $c$ ) and robot ( $b$ and d). In the simulation, the green dots identify the head and the blue line represents the trajectory of center of mass. In figure $\mathrm{b}$ and $\mathrm{d}$, the module connected to hanging tail (not making contact) indicates the hind module.

\section{B. Periodic Ground Contacts}

In legged systems, robots and animals repeatedly make and break contact with the environment. We introduce a binary contact state variable, $\boldsymbol{C}$, that describes the contact states: 0 (no contact, therefore no forces) or 1 (full contact). Thus, the local connection becomes a function of both shape variables and contact states:

$$
\xi=A(r, C) \dot{r} \text {. }
$$

In our case, $\boldsymbol{\xi}=\left[\begin{array}{lll}\xi_{x} & \xi_{y} & \xi_{\theta}\end{array}\right]^{T}$ (Fig. 1b) denotes the body velocity in forward, lateral and rotational direction, $\boldsymbol{C}=\left[\begin{array}{llll}c_{1} & c_{2} & c_{3} & c_{4}\end{array}\right]^{T}$ denotes the leg contact states of four limbs (see Fig. 1k) and $\boldsymbol{r}=\left[\begin{array}{lllll}\alpha_{b} & \beta_{1} & \beta_{2} & \beta_{3} & \beta_{4}\end{array}\right]^{T}$ denotes the shape variables (joint angles, see Fig. 1p).

We assume that the contact variable $C$ and the shape variable $\beta_{i}$ can all be written as a function of phase $\tau$, i.e.,

$$
c_{i}=F_{i}(\tau), \beta_{i}=f_{i}(\tau) i=1,2,3,4 .
$$

The actual derivation of the functions $F_{i}$ and $f_{i}$ will be introduced in section $\mathrm{V}$. With these functions in hand, the shape space can consist of two variables $\alpha_{b}$ and $\tau$, the back bending angle and the phase, forming the reduced shape variable $\boldsymbol{\Omega}=\left[\alpha_{b} \tau\right]^{T}$. We can thus rewrite Eq. $(4)$ as:

$$
\boldsymbol{\xi}=\boldsymbol{A}(\boldsymbol{r}, \boldsymbol{C}) \dot{\boldsymbol{r}}=\boldsymbol{A}(\boldsymbol{\Omega})\left[\begin{array}{lr}
1 & 0 \\
0 & d f_{1}(\tau) / d \tau \\
0 & d f_{2}(\tau) / d \tau \\
0 & d f_{3}(\tau) / d \tau \\
0 & d f_{4}(\tau) / d \tau
\end{array}\right] \dot{\boldsymbol{\Omega}}=\boldsymbol{A}^{\prime}(\boldsymbol{\Omega}) \dot{\boldsymbol{\Omega}}
$$

where $\boldsymbol{\Omega}=[\tau, \alpha]^{T}, \boldsymbol{A}^{\prime}(\boldsymbol{\Omega})$ is the new local connection relating the reduced shape velocity $\dot{\boldsymbol{\Omega}}$ to body velocity $\boldsymbol{\xi}$. Since $\tau$ is cyclic, the shape space is now cylindrical.

\section{Granular Resistive Force Theory}

Granular resistive force theory (RFT) [56, 37, 49] has been exploited to model the animals and robots locomotion in contact with granular materials. Prior work [23] has shown that numerically deriving local connections and height functions using granular RFT can predict effective movements in granular media.

Granular RFT allows us to decompose the resistive force experienced on an infinitesimally small portion of a locomotive intruder into two components: thrust and drag. The reaction force applied on the entire system is computed as,

$$
F=\int\left(d F_{\|}+d F_{\perp}\right),
$$

where $\boldsymbol{F}_{\|}$and $\boldsymbol{F}_{\perp}$ respectively denote forces parallel and perpendicular to a segment. During intrusions in granular media at slow speeds, the attack angle, which can be computed from the body velocity, determines $\boldsymbol{F}_{\|}$and $\boldsymbol{F}_{\perp} . \boldsymbol{F}_{\|}$and $\boldsymbol{F}_{\perp}$ are independent from the magnitude of the velocity [56]. More details regarding granular RFT can be found in [56].

By assuming that the motions of quadrupeds in granular material are quasi-static [21], we can consider the total net force applied on the system is zero.

$$
F=\int\left(d F_{\|}+d F_{\perp}\right)=0,
$$

The body velocity is the only unknown in the above equation. It can be computed by solving for Eq. 8 , with a 


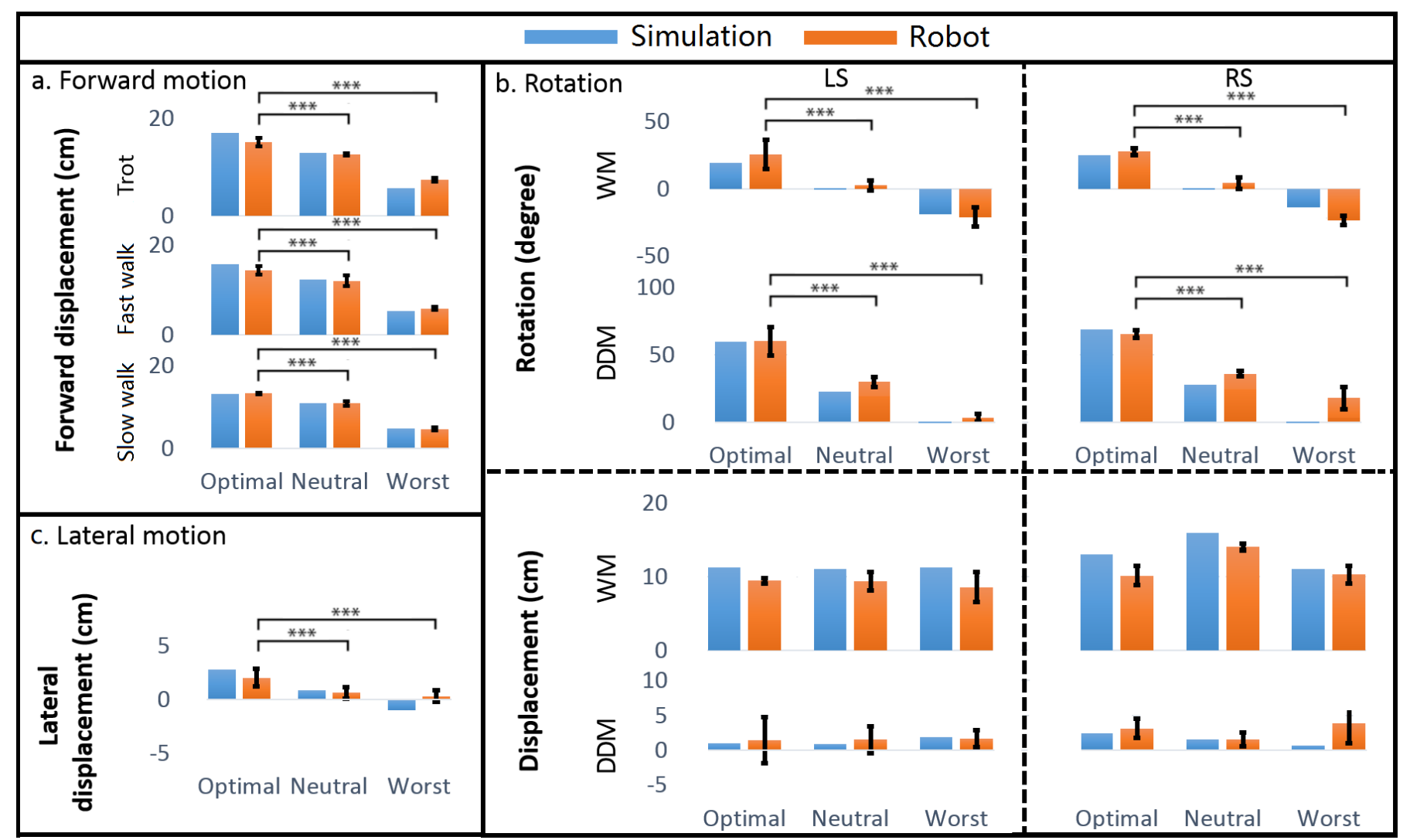

Fig. 6. Experiment results of RFT simulations (blue) and robot experiments (orange) of (a) forward, (b) rotational and (c) lateral gaits, showing close agreement between simulations and experiments. Each gait is tested for 3 experiment trials, each containing at least 3 gait periods. The "optimal", "neutral" and "worst" respectively represent optimal back bending, no back bending and worst back bending. Means and standard deviations can be found in the supplement materials. We indicate statistically significant [52] improvement, when comparing the 'optimal'-'neutral', as well as 'optimal'-'worst' gaits ( ${ }^{*}: p<.05$, ${ }^{* *}: p<.01,{ }^{* * *} p<.001$ ). For LS (lateral sequence), RS (rotary sequence), DDM (differential drive machine) and WM (walking machine), we show both displacement and rotation values for completeness only: back bending is optimized wrt. rotation only, and displacement changes are not meaningful.

given shape velocity $\dot{\boldsymbol{\Omega}}$. In our implementation, we compute the root of Eq. (8) using the MATLAB function fminsearch.

With above assumptions, the local connection of a quadrupedal robot moving in granular media can be approximated by numerically calculating displacements in the body frame. We refer readers to [21] for detailed numerical calculations. This numerically-determined local connection can be used to plot connection vector fields and height functions.

\section{Gait design}

We choose the path in the shape space to be represented by truncated Fourier series [2]. Also, we set a constraint on the magnitude of back bending as the joint angle limit:

$$
\max _{\tau} \mid\left(\alpha_{b}(\tau) \mid=\Gamma_{\alpha}\right. \text {. }
$$

We can now maximize the area under the curve by optimizing (by MATLAB's fminsearch function) over the parameters $a_{n}$ and $b_{n}$ which parameterize the path. We find that a path in the shape space is well approximated by the first two terms in the series (see Fig. 4):

$$
\alpha_{b}=\sum_{n=1,2} a_{n} \cos \left(n \tau+b_{n}\right) .
$$

\section{EXPERIMENTAL SETUP}

We subscribe to a philosophy called robotphysics [1] in which a robot is used to verify and explore models of physics, in this case motion of our system in a granular medium. We built a quadrupedal robot (Fig. 119) and tested its performance on granular media. This open-loop, servo-driven, 3D-printed robot (450 g, $40 \mathrm{~cm}$ long) has four legs and an actuated back. Each leg has two servo motors to control its vertical position and the step size. A joint in the middle of the body controls horizontal bending.

Using a fully-automated setup [45], we experimentally and systematically tested the importance of coordination between back bending and leg movements on the bed filled with $\sim 1 \mathrm{~mm}$ diameter poppy seeds. Each gait is tested for three experiment trails and each trials have at least three gait periods. The robot experiments and RFT simulation executing the same gaits are compared in Fig 6.

Tilting actuators set the inclination angle of the bed to zero at the beginning of each experiment. The robot then executes a programmed set of movements to walk on the loosely-packed poppy seeds. Throughout the experiment, four Optitrack Flex13 cameras record the positions of infraredreflective markers on the robot. At the end of each experiment, the robot's final position is identified. A 3-axis motor system moves to the robot, picks it up and sends it back to the starting position. An air-fluidized bed erases the footprints and allows the seeds to be reset into a loosely-packed state [36]. 

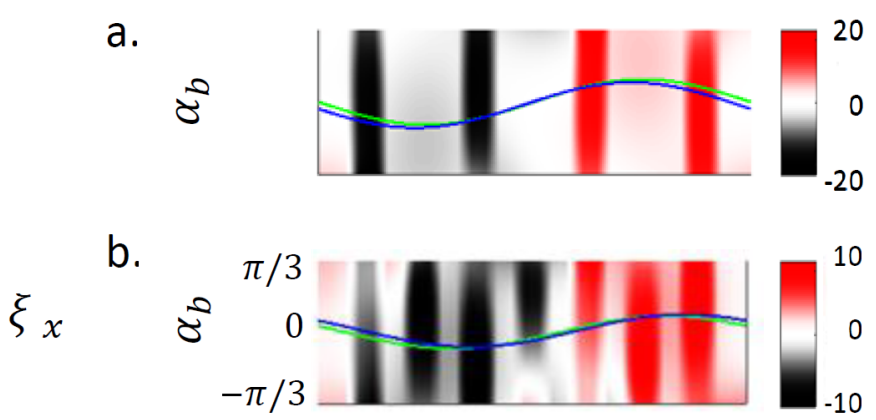

C.

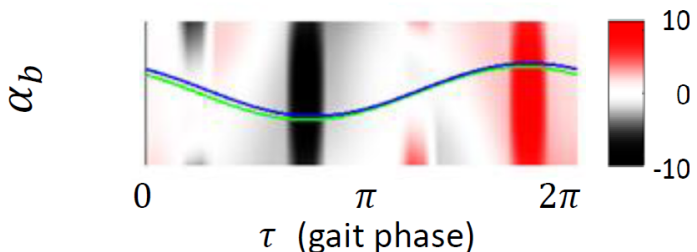

Fig. 7. Height function for the experimentally-measured gait for (a) trot (b) fast walk and (c) slow walk, with experimental gait (blue) and geometric mechanics predicted gait (green) in forward direction overlaid. All the panels have the same body angle range as in the middle panel.

\section{RESUlts}

\section{A. Forward Motion}

1) Leg movement: We test three typical leg movements: the slow walk gait, the fast walk gait and the trot gait from the Hildebrand analysis [24], where the pattern of the symmetrical gaits is expressed with only two parameters: lateral leg phase shift (the fraction of the step cycle that hind limbs lead the fore limbs on the same side) and duty factor (the fraction of the step cycle where the foot is on the ground). The duty factors for the three gaits are all 0.75 , and the lateral leg phase shits are: $0.25,0.375$, and 0.5 .

The trot gait (lateral leg phase shift $=0.5$ ) is a gait with diagonally paired leg movement. The fore right leg is always in phase with the hind left leg; while fore left leg is always in phase with the hind right leg. The slow walk gait (lateral leg phase shift $=0.25$ ) is a gait with evenly spaced leg lifting following the sequence fore right, hind left, fore left, hind right (defined as lateral sequence [24]). The fast walk (lateral leg phase shift $=0.375$ ) is another four-beat gait following the lateral sequence. Unlike the slow walk gait, there is an overlap of the aerial phase between fore right and hind left legs(as well as fore left and hind right). The detailed descriptions of these gaits are presented in Fig. 3a. Interestingly, the overlaps in diagonal leg activation increases from slow walk, fast walk to trot, which leads to higher speed.

2) Gait design: For each leg movement, we calculate the forward height functions (Fig. 4 ). Here, we set $\Gamma_{\alpha}=\frac{\pi}{3}$.

Recall that for height functions on cylinders, the displacement of a gait is the volume underneath the gait path drawn on the parameterization of the cylinder. The gait path with most maximum volume ("optimal" gait) underneath should be the gait with largest forward stride displacement per gait cycle. We observe that the "optimal" back bending gaits are dominated by the first component of the Fourier Series, meaning that leg movements and back bending follow the same frequency.
We also identify the worst back bending coordination with minimal volume underneath in height function ("worst" gait). We test the "neutral" gait with constant straight back.

We verify our gaits with RFT simulations and robot experiments across granular materials. Both RFT simulations and robot experiments suggest that the proper back-leg coordination can improve the forward stride displacement, while the improper phasing can lead to ineffective forward gait. Simulation and experiment data are presented in Fig. 63.

3) Animal comparison: We use the fire salamander (Salamandra salamandra) as a model animal and investigate if the animals use back bending kinematics which optimize forward progress for a given stepping pattern. For these experiments, individual animals walk along a straight trackway filled with a sand-like substrate (300-um glass particles). Three cameras are positioned around the trackway and recorded synchronized videos at 120 FPS. At least three gait periods were recorded in each experiment. Limb positions, body angles, and footfall timing are manually extracted from each recording. According to the limb positions and footfall timing, we selected three typical salamander motion videos (each contains at least three gait periods of animal motion), which coorespond to "trot" (duty factor $0.75 \pm 0.03$, lateral leg phase shift $0.25 \pm 0.05$ ), "fast walk" (duty factor $0.76 \pm 0.04$, lateral leg phase shift $0.36 \pm 0.02$ ) and "slow walk" gaits (duty factor $0.73 \pm 0.1$, lateral leg phase shift $0.50 \pm 0.02$ ). We fitted the animal body angles with first two terms of Fourier Series as in Eq.10.

For each leg movement, we calculate the height function in Fig. 7. We plotted the fitted animal body bending on cooresponding height functions. Setting the body-bending amplitude, $\Gamma_{\alpha}$, to match experimental data, we design the back bending gait to coordinate with leg movement. The resulting predicted gait and animal gait are in good agreement (Fig. 7).

Note that the RFT for animals (300-um glass beads) is slightly different from the RFT for robots (poppy seeds). We therefore used the parameters from the animal data [4, 13] to create the height functions .

\section{B. Rotational motion}

1) Leg movement: While the lateral footfall sequence (FRHL-FL-HR) is widely used in forward walking, other sequences can produce other motions. Hirose et al. [25] introduced rotary footfall sequence (FR-HR-HL-FL) that favors counterclockwise turning. We investigate back bending coordination in both lateral sequence (LS) and rotary sequence (RS).

In addition to the footfall sequence, the amplitude of leg movement can also be important during rotation [19]. Whereas identical leg amplitudes (walking machines, WM) will lead to positive forward translation without rotation, differentia ${ }^{1}$ in lateral leg amplitudes (differential drive machine, DDM) will lead to counterclockwise $(\mathbf{C C W})$ or clockwise $(\mathbf{C W})$ rotations, depending on the relative amplitudes of each leg. We call CCW rotation as the positive direction and study $\mathrm{CCW}$ rotation in

\footnotetext{
${ }^{1}$ differential here means difference like in a differential drive car, as opposed to difference like in a derivative
} 
DDM. We investigate what role the back bending will play in rotation for both WM and DDM. The detailed descriptions of leg movements are presented in Fig. 3 b.

2) Gait design: For each leg movement in Fig. 3p, we calculate the rotational height function (Fig. 4p). Note that we set $\Gamma_{\alpha}$ to be $\frac{\pi}{3}$ in this subsection.

From the rotational height function in Fig. $4 \mathrm{~b}$.1, we observe that the rotational displacement is maximized when the frequency of the back bending is twice that of the leg movement. Given the same leg movement, in previous subsection we conclude the forward displacement is maximized when the back bending and the leg movement follow the same frequency. Thus, the proper back bending can either improve the forward locomotion performance, or rotate the head orientation while moving forward. The comparison is illustrated in Fig. 5. Essentially, the back bending expands the target position space. Further discussions on the role of back bending can be found in the section VI.

For WM, the net leg movement will lead to forward displacement without rotation. Therefore, with the inclusion of back bending, we expect $\mathrm{CW}$ or $\mathrm{CCW}$ rotation (determined by the phase of back bending) plus forward displacement. The gait with maximal volume underneath ("optimal" gait) is that with the most $\mathrm{CCW}$ rotation per circle. As a result, the robot simultaneously changes its head direction and moves forward, which we call the walking turn. Note that the converse is also true: the gait with minimal volume underneath ("worst" gait) is that with least $\mathrm{CCW}$ (i.e., most $\mathrm{CW}$ ) rotation.

For DDM, the net leg movement will lead to CCW rotations without displacement. Thus, with inclusion of back bending, we can expect more rotation with no displacement. The back bending with maximum ("optimal" gait) volume underneath height function will increase the CCW rotation. Since the net displacements are negligible for differential drive machine, the robot essentially rotates while without translating (in place turn). In the converse case, the gait with minimal volume ("worst" gait) underneath is the gait with least counterclockwise (i.e., most clockwise) rotation. However, the rotation by the back bending cancels out the rotation by the leg movement, leading to ineffective rotation.

We verify our theory with RFT simulations and robot experiments across granular materials. For reference, we also test the neutral gait with constant straight back. The data is presented in Fig. 6b.

Finally, both RFT simulations and robot experiments suggest that the rotary footfall sequences (RS) have larger rotational angles than lateral footfall sequences (LS), which is consistent with Hirose et al. [25].

\section{Lateral motion}

1) Leg movement: Hirose et al. [25] introduced the footfall patterns that produce lateral motion. To achieve better lateral displacement, we coupled fore right leg to be in phase of hind right leg, fore left leg to be in phase of hind left leg.

We test the left sideways motion. The converse should be the same for right sideways motion. The leg movements follow the assumption that the leg is at its left joint angle limit at the end of aerial phase; and the leg is at its right joint angle limit at the beginning of aerial phase. The detailed description of leg movement is shown in Fig. 3r.

2) Gait design: For each leg movement, we calculate lateral height functions (Fig. 4p). In this subsection, we set $\Gamma_{\alpha}=\frac{\pi}{3}$.

The back-leg coordination with the positive volume underneath can lead to the most left lateral move. We verifiy our gaits with RFT simulations and robot experiments on granular materials. For reference, we also test the neutral gait with constant straight back. The data is listed in Fig. 6.

\section{CONCLUSION}

\section{A. Whole body motion with back bending coordination}

We apply and extend geometric mechanics to design gaits of back bending to coordinate with leg movements. We prescribed the leg movements based on existing research on legged systems and advance the current legged locomotion by adding back bending coordination. Geometric mechanics allows us to intuitively design and analyze gait paths for different modes of quadrupedal motions. In contrast to the feedback control algorithms in CPG, our gait design algorithms do not require prior knowledge of gait shapes. However, we observe that our approach can be used as a base for CPGbased approaches. That is, our gait design process can be used to generate and optimize gait trajectories, which can then be tracked online by a set of coupled oscillators.

In addition, our methods of coordinating back bending can extend to other morphologies. Our future work will consider other types of bends, which may be represented by modal functions, along with other morphologies that may benefit from back bending.

\section{B. Locomotion performance improvement}

In this paper, we discussed how proper coordination in back bending and limb movement can improve forward, rotational and lateral locomotion. Our theory is verified by RFT simulation and robot experiments. Furthermore, we also observed our calculated back bending coordinations to improve forward displacements are close to those from animal locomotion experiments. Our future work will include a systematical study of animal whole-body coordination and evaluate it in terms of speed, stability and cost of transport.

Back bending can also result in different modes of locomotion. We showed that the proper designed back bending gait paths can either increase forward displacement or add rotation (both clockwise and counterclockwise) to forward displacement. This is an example of how back bending can expand the target position space. Future work will include the reference to biological data and even the application of machine learning algorithms to further expand the target position space. 


\section{REFERENCES}

[1] Jeffrey Aguilar, Tingnan Zhang, Feifei Qian, Mark Kingsbury, Benjamin McInroe, Nicole Mazouchova, Chen Li, Ryan Maladen, Chaohui Gong, Matt Travers, et al. A review on locomotion robophysics: the study of movement at the intersection of robotics, soft matter and dynamical systems Reports on Progress in Physics, 79 (11):110001, 2016.

[2] R. McN. Alexander. The Gaits of Bipedal and Quadrupedal Animals The International Journal of Robotics Research, 3(2):49-59, 1984. doi: 10.1177/ 027836498400300205.

[3] R. Altendorfer, N. Moore, H. Komsuoglu, M. Buehler, H.B. Brown, D. McMordie, U. Saranli, R. Full, and D.E. Koditschek. RHex: A Biologically Inspired Hexapod Runner Autonomous Robots, 11(3):207-213, Nov 2001. ISSN 1573-7527. doi: 10.1023/A:1012426720699. URL https://doi.org/10.1023/A:1012426720699.

[4] Yasemin Ozkan Aydin, Baxi Chong, Chaohui Gong, Jennifer M Rieser, Jeffery W Rankin, Krijn Michel, Alfredo G Nicieza, John Hutchinson, Howie Choset, and Daniel I Goldman. Geometric Mechanics Applied to Tetrapod Locomotion on Granular Media. In Conference on Biomimetic and Biohybrid Systems, pages 595-603. Springer, 2017.

[5] Shaoping Bai, K. H. Low, G. Seet, and T. Zielinska. A new free gait generation for quadrupeds based on primary/secondary gait In Proceedings 1999 IEEE International Conference on Robotics and Automation (Cat. No.99CH36288C), volume 2, pages 1371-1376 vol.2, 1999. doi: 10.1109/ROBOT.1999.772552.

[6] Robert W Batterman. Falling cats, parallel parking, and polarized light. Studies in History and Philosophy of Science Part B: Studies in History and Philosophy of Modern Physics, 34(4):527-557, 2003.

[7] Z. Bien, M. G. Chun, and H. S. Son. An optimal turning gait for a quadruped walking robot In Intelligent Robots and Systems '91. 'Intelligence for Mechanical Systems, Proceedings IROS '91. IEEE/RSJ International Workshop on, pages 1511-1514 vol.3, Nov 1991. doi: 10.1109/IROS.1991.174725.

[8] Martin Buehler, R Battaglia, A Cocosco, Geoff Hawker, J Sarkis, and K Yamazaki. SCOUT: A simple quadruped that walks, climbs, and runs In Robotics and Automation, 1998. Proceedings. 1998 IEEE International Conference on, volume 2, pages 1707-1712. IEEE, 1998.

[9] Martin Buehler, A Cocosco, K Yamazaki, and $\mathrm{R}$ Battaglia. Stable open loop walking in quadruped robots with stick legs. In Robotics and Automation, 1999. Proceedings. 1999 IEEE International Conference on, volume 3, pages 2348-2353. IEEE, 1999.

[10] Jorge G. Cham, Jonathan K. Karpick, and Mark R. Cutkosky. Stride Period Adaptation of a Biomimetic Running Hexapod. The International Journal of Robotics Research, 23(2):141-153, 2004. doi: 10.1177/
0278364904041323.

[11] S. Chernova and M. Veloso. An evolutionary approach to gait learning for four-legged robots. In 2004 IEEE/RSJ International Conference on Intelligent Robots and Systems (IROS) (IEEE Cat. No.04CH37566), volume 3, pages 2562-2567 vol.3, Sept 2004. doi: 10.1109/IROS. 2004.1389794.

[12] D. J. Cho, J. H. Kim, and D. G. Gweon. Optimal turning gait of a quadruped walking robot Robotica, 13(6): 559564, 1995. doi: 10.1017/S0263574700018634.

[13] Baxi Chong, Jennifer Rieser, Yunjin Wu, Haosen Xing, Howie Choset, Daniel Goldman, et al. The importance of body-limb coordination in a walking tetrapod Bulletin of the American Physical Society, 2018.

[14] A. Crespi, K. Karakasiliotis, A. Guignard, and A. J. Ijspeert. Salamandra Robotica II: An Amphibious Robot to Study Salamander-Like Swimming and Walking Gaits IEEE Transactions on Robotics, 29(2):308-320, April 2013. ISSN 1552-3098. doi: 10.1109/TRO.2012. 2234311.

[15] Holk Cruse. What mechanisms coordinate leg movement in walking arthropods? Trends in Neurosciences, 13(1): 15 - 21, 1990. ISSN 0166-2236.

[16] Jin Dai, Hossein Faraji, Chaohui Gong, Ross L Hatton, Daniel I Goldman, and Howie Choset. Geometric Swimming on a Granular Surface. In Robotics: Science and Systems, 2016.

[17] Jonas Degrave, Michal Burm, Pieter-Jan Kindermans, Joni Dambre, and Francis wyffels. Transfer learning of gaits on a quadrupedal robot Adaptive Behavior, 23(2): 69-82, 2015. doi: 10.1177/1059712314563620.

[18] I. Delvolve, P. Branchereau, R. Dubuc, and J.-M. Cabelguen. Fictive rhythmic motor patterns induced by NMDA in an in vitro brain stemspinal cord preparation from an adult urodele Journal of Neurophysiology, 82 (2):1074-1077, 1999.

[19] Gregory Dudek and Michael Jenkin. Computational principles of mobile robotics Cambridge university press, 2010.

[20] Peter Eckert, Alexander Spröwitz, Hartmut Witte, and Auke Jan Ijspeert. Comparing the effect of different spine and leg designs for a small bounding quadruped robot. In Robotics and Automation (ICRA), 2015 IEEE International Conference on, pages 3128-3133. IEEE, 2015.

[21] Chaohui Gong, Daniel I Goldman, and Howie Choset. Simplifying Gait Design via Shape Basis Optimization. In Robotics: Science and Systems, 2016.

[22] Chaohui Gong, Julian Whitman, Jaskaran Grover, Baxi Chong, Richard Ren, and Howie Choset. Geometric motion planning for systems with toroidal and cylindrical shape spaces (submitted). In Dynamic Systems and Control Conference, 2018.

[23] Ross L Hatton, Yang Ding, Howie Choset, and Daniel I Goldman. Geometric visualization of self-propulsion in a complex medium. Physical review letters, 110(7): 
078101, 2013.

[24] Milton Hildebrand. Symmetrical gaits of horses science, 150(3697):701-708, 1965.

[25] Shigeo Hirose, Hidekazu Kikuchi, and Yoji Umetani. The standard circular gait of a quadruped walking vehicle. Advanced Robotics, 1(2):143-164, 1986.

[26] P. Holmes, R. J. Full, D. Koditschek, and J. Guckenheimer. The dynamics of legged locomotion: Models, analyses, and challenges. Siam Review, 48(2):207-304, 2006.

[27] Auke Jan Ijspeert. A connectionist central pattern generator for the aquatic and terrestrial gaits of a simulated salamander Biological Cybernetics, 84(5):331-348, Apr 2001. ISSN 1432-0770. doi: 10.1007/s004220000211.

[28] Auke Jan Ijspeert, Alessandro Crespi, Dimitri Ryczko, and Jean-Marie Cabelguen. From Swimming to Walking with a Salamander Robot Driven by a Spinal Cord Model Science, 315(5817):1416-1420, 2007. ISSN 0036-8075. doi: 10.1126/science.1138353.

[29] Neri Kafkafi and Ilan Golani. A traveling wave of lateral movement coordinates both turning and forward walking in the ferret. Biological Cybernetics, 78(6):441-453, Jul 1998. ISSN 1432-0770. doi: 10.1007/s004220050448.

[30] Scott D Kelly and Richard M Murray. Geometric phases and robotic locomotion. Journal of Field Robotics, 12 (6):417-431, 1995.

[31] Min Sub Kim and William Uther. Automatic Gait Optimisation for Quadruped Robots. In In Australasian Conference on Robotics and Automation, 2003.

[32] Shoshichi Kobayashi and Katsumi Nomizu. Foundations of differential geometry, volume 1. Interscience publishers New York, 1963.

[33] N. Kohl and P. Stone. Policy gradient reinforcement learning for fast quadrupedal locomotion. In Robotics and Automation, 2004. Proceedings. ICRA '04. 2004 IEEE International Conference on, volume 3, pages 2619-2624 Vol.3, April 2004. doi: 10.1109/ROBOT. 2004.1307456.

[34] J Zico Kolter, Mike P Rodgers, and Andrew Y Ng. A control architecture for quadruped locomotion over rough terrain In Robotics and Automation, 2008. ICRA 2008. IEEE International Conference on, pages 811-818. IEEE, 2008.

[35] Karl Frederick Leeser. Locomotion experiments on a planar quadruped robot with articulated spine. $\mathrm{PhD}$ thesis, Massachusetts Institute of Technology, 1996.

[36] Chen Li, Paul B Umbanhowar, Haldun Komsuoglu, Daniel E Koditschek, and Daniel I Goldman. Sensitive dependence of the motion of a legged robot on granular media Proceedings of the National Academy of Sciences, 106(9):3029-3034, 2009.

[37] Chen Li, Tingnan Zhang, and Daniel I Goldman. A terradynamics of legged locomotion on granular media. Science, 339(6126):1408-1412, 2013.

[38] Jerrold E Marsden. Geometric foundations of motion and control In Motion, Control, and Geometry: Proceedings of a Symposium, Board on Mathematical Science, National Research Council Education, National Academies Press, Washington, DC, 1997.

[39] Jerrold E Marsden and Tudor S Ratiu. Introduction to mechanics and symmetry: a basic exposition of classical mechanical systems volume 17. Springer Science \& Business Media, 2013.

[40] R. B. Mcghee and G. I. Iswandhi. Adaptive Locomotion of a Multilegged Robot over Rough Terrain IEEE Transactions on Systems, Man, and Cybernetics, 9(4): 176-182, April 1979. ISSN 0018-9472. doi: 10.1109/ TSMC.1979.4310180.

[41] Jim Ostrowski and Joel Burdick. The geometric mechanics of undulatory robotic locomotion The international journal of robotics research, 17(7):683-701, 1998.

[42] Dai Owaki, Takeshi Kano, Ko Nagasawa, Atsushi Tero, and Akio Ishiguro. Simple robot suggests physical interlimb communication is essential for quadruped walking Journal of The Royal Society Interface, 10(78), 2013. ISSN 1742-5689. doi: 10.1098/rsif.2012.0669.

[43] L. R. Palmer and D. E. Orin. Attitude Control of a Quadruped Trot While Turning. In 2006 IEEE/RSJ International Conference on Intelligent Robots and Systems, pages 5743-5749, Oct 2006. doi: 10.1109/IROS.2006. 282381.

[44] Michael JD Powell. An efficient method for finding the minimum of a function of several variables without calculating derivatives The computer journal, 7(2):155162, 1964.

[45] F. Qian, K. Daffon, T. Zhang, and D. I. Goldman. An automated system for systematic testing of locomotion on heterogeneous granular media. In Proceedings of the 16th International Conference on Climbing and Walking Robots (CLAWAR), pages 1-8, 2013.

[46] S. Rossignol, R. Dubuc, and J.-P. Gossard. Dynamic sensorimotor interactions in locomotion Physiological reviewsw, 86(1):89-154, 2006.

[47] Uluc Saranli, Martin Buehler, and Daniel E. Koditschek. RHex: A Simple and Highly Mobile Hexapod Robot The International Journal of Robotics Research, 20(7): 616-631, 2001. doi: 10.1177/02783640122067570.

[48] Alfred Shapere and Frank Wilczek. Geometry of selfpropulsion at low Reynolds number. Journal of Fluid Mechanics, 198:557-585, 1989.

[49] Sarah S Sharpe, Stephan A Koehler, Robyn M Kuckuk, Miguel Serrano, Patricio A Vela, Joseph Mendelson, and Daniel I Goldman. Locomotor benefits of being a slender and slick sand swimmer. Journal of Experimental Biology, 218(3):440-450, 2015.

[50] Theo H Smit. The use of a quadruped as an in vivo model for the study of the spine-biomechanical considerations European Spine Journal, 11(2):137-144, 2002.

[51] J. A. Smith and I. Poulakakis. Rotary gallop in the untethered quadrupedal robot scout II In 2004 IEEE/RSJ International Conference on Intelligent Robots and Systems (IROS) (IEEE Cat. No.04CH37566), volume 3, 
pages 2556-2561 vol.3, Sept 2004. doi: 10.1109/IROS. 2004.1389793.

[52] Richard C Sprinthall and Stephen T Fisk. Basic statistical analysis Prentice Hall Englewood Cliffs, NJ, 1990.

[53] Peter Stone, Kurt Dresner, Selim T Erdogan, Peggy Fidelman, Nicholas K Jong, Nate Kohl, Gregory Kuhlmann, Ellie Lin, Mohan Sridharan, Daniel Stronger, et al. UT Austin Villa 2003: A new RoboCup four-legged team 2003.

[54] M. Wheatley, K. Jovanovic, R. B. Stein, and V. Lawson. The activity of interneurons during locomotion in the in vitro necturus spinal cord Journal of Neurophysiology, 71(6):2025-2032, 1994.

[55] Frank Wilczek and Alfred Shapere. Geometric phases in physics, volume 5. World Scientific, 1989.

[56] Tingnan Zhang and Daniel I Goldman. The effectiveness of resistive force theory in granular locomotion a. Physics of Fluids, 26(10):101308, 2014.

[57] Matt Zucker, Nathan Ratliff, Martin Stolle, Joel Chestnutt, J Andrew Bagnell, Christopher G Atkeson, and James Kuffner. Optimization and learning for rough terrain legged locomotion The International Journal of Robotics Research, 30(2):175-191, 2011. 